\title{
Interdisciplinarité et représentation de la complexité des systèmes socio-écologiques : recherches sur la zone atelier Armorique ${ }^{\star}$
}

\author{
Jacques Baudry ${ }^{1, *}$, Audrey Alignier ${ }^{1}$ et Zahra Thomas ${ }^{2}$ \\ ${ }^{1}$ Écologie, INRA, UMR BAGAP, Rennes, France \\ ${ }^{2}$ Hydraulique, hydrologie, Agrocampus Ouest, INRA, UMR SAS, Rennes, France
}

Le développement des politiques publiques en matière d'environnement nécessite des bases scientifiques pour en définir les modes d'action. Prévoir, évaluer, autant que possible, les conséquences environnementales constituent un enjeu majeur. Lorsque ces politiques deviennent plus globales et non plus sectorielles (des réserves pour la faune et la flore, qualité de l'eau, etc.), les problèmes à traiter deviennent plus complexes. Cette complexité s'exprime au travers des interactions entre les processus écologiques, auxquelles s'ajoutent les interactions entre les acteurs des territoires et la diversité de leurs actions sur ces territoires et leurs impacts sur les ressources.

Ces questions ont donné lieu au développement de recherches sur les systèmes socioécologiques qui prennent explicitement et fonctionnellement en compte les interactions entre les activités humaines et les processus écologiques (Carpenter et al., 2009; De Longe et al., 2016; Head et Xiang, 2016).

Les acteurs de ces politiques publiques sont alors confrontés à deux grands types d'enjeux: la nature des informations scientifiques disponibles et la mise en place de dispositifs de recherche adéquats pour traiter de cette complexité.

\section{Les informations scientifiques disponibles pour la décision publique}

Le courant de pensée dominant en matière d'environnement aborde ces enjeux comme un problème d'ingénierie classique. Il suffit de connaître les causes

\footnotetext{
‡ Voir dans ce numéro le texte d'introduction de Marcel Jollivet.

* Auteur correspondant : jacques.baudry@inra.fr
}

pour maîtriser les processus écologiques. Même compliquée, la combinaison de l'observation, de l'expérimentation et de la modélisation doit permettre de comprendre et d'agir. C'est la base du développement des politiques basées sur des faits avérés ${ }^{1}$. Cette approche puise ses origines dans la recherche médicale (Laurent et al., 2009). Afin de construire des politiques environnementales, plusieurs pays européens disposent de services ministériels chargés d'établir des documents sur des faits avérés (comme les Pays-Bas, le Royaume-Uni). C'est aussi une position adoptée par la commission européenne. Depuis plusieurs années, un groupe de biologistes de la conservation produit des documents selon cette méthodologie ${ }^{2}$. La force de cette démarche est de mettre en évidence des relations de cause à effet, de préférence avec un dispositif expérimental le mieux contrôlé possible et un essai randomisé. Mais sa faiblesse est de ne pouvoir prendre en compte toute la complexité du système étudié. Cette dernière ne peut être étudiée par des expérimentations, même multifactorielles. Par définition, un système complexe est un système plein

\footnotetext{
${ }^{1}$ En France, la Fondation pour la recherche sur la biodiversité (FRB) définit cette démarche basée sur les revues systématiques, qui « ont pour ambition de tenir compte de l'ensemble des connaissances disponibles pour répondre à un besoin de connaissances présenté sous la forme d'une question structurée [...] Il s'agit (de fournir) des arguments étayés qui montrent l'existence de faits avérés ou, au contraire, soulignent les lacunes de connaissances et les priorités de recherche». Les meilleurs faits avérés sont ceux qui sont issus d'essais contrôlés randomisés. Il n'est par contre pas possible de randomiser des essais quand on veut tester les effets d'une politique en interaction avec une diversité de pratiques, comme dans le cas de l'environnement.

${ }^{2}$ http://conservationevidence.com/
} 
d'incertitudes dues aux interactions non linéaires et aux multiples rétroactions (Liu et al., 2007).

Dans un système expérimental, la production de faits avérés permet de définir une chaîne classique qui va de l'analyse d'un problème à sa compréhension et permet la définition de règles de gestion. Ce sont des systèmes maîtrisés ou maîtrisables (tamed systems). Un système complexe ne peut être géré de cette façon (Hancock, 2004). Depuis de nombreuses années, les sciences de la gestion d'entreprise et de l'aménagement ont développé le concept de "problème malicieux » (wicked problem). On parle de «problème malicieux » lorsque l'incertitude sur les conditions futures (environnementales, économiques et sociales) et les différences dans les valeurs sociologiques et économiques rendent impossible la définition d'une solution optimale à toutes les échelles et dans tous les domaines. Ce concept a été développé dès les années 1970 par (Rittel et Webber, 1973). Dans la seconde moitié $\mathrm{du} \mathrm{xx}^{\mathrm{e}}$ siècle, les décideurs et les gestionnaires ont pensé que les problèmes de perte de biodiversité étaient des problèmes maîtrisables. La création d'aires protégées pour la conservation de la biodiversité a été la base de ces politiques en éloignant les activités humaines des processus écologiques. Nous savons maintenant que les problèmes environnementaux ne sont plus envisagés de cette manière. Ce sont des problèmes liés à des systèmes socioécologiques couplant systèmes sociaux et système naturels. Ces systèmes sont complexes. Les apports de la science à la décision publique ne peuvent donc être linéaires, ni à sens unique : il faut comprendre les modalités d'une action pour en relier les effets sur des processus écologiques liés à des problèmes environnementaux.

\section{Les dispositifs de recherche adéquats pour traiter la complexité : les zones atelier}

Les zones atelier sont des dispositifs à long terme de recherche interdisciplinaire mis en place par le CNRS en 2000 et soutenu par l'Inra ${ }^{3}$. L'objectif est d'articuler différentes approches (écologiques, géographiques, juridiques, agronomiques, sociales, etc.) sur un territoire donné pour analyser les dynamiques environnementales et sociales. Actuellement le réseau français compte 12 zones atelier. Il est intégré dans le réseau européen et international des sites de recherche à long terme en écologie ${ }^{4}$. Parmi ces réseaux internationaux traitant d'environnement, le fait d'être basé sur un site commun est une spécificité des sites de recherche à long terme en

\footnotetext{
${ }^{3} \mathrm{http} / / /$ www.za-inee.org/

${ }^{4}$ International Long Term Ecological Research, https://www. ilternet.edu/
}

écologie (Maass et al., 2016). Sans entrer dans le détail de l'histoire, il faut dire que les zones atelier sont le produit du réseau LTER des États-Unis et d'une tradition de recherche interdisciplinaire alliant sciences de la nature et sciences sociales en France (Jollivet, 1992). Ceci a permis d'emblée la construction de programmes interdisciplinaires.

La zone atelier Armorique est issue de projets de recherche en écologie du paysage sur le bocage associant de multiples laboratoires rennais dès 1993. Dans le cadre d'un programme du ministère chargé de l'Environnement sur les structures linéaires boisées, le conseil scientifique avait décidé que les différentes équipes devaient se concentrer sur deux sites, l'un en Bretagne, l'autre dans les Alpes. Labellisée en 2002, la zone atelier Armorique est un des dispositifs de l'Observatoire des sciences de l'univers de Rennes (OSUR) ${ }^{5}$. Elle comporte une partie rurale, notamment au nord de l'Ille-et-Vilaine, le site historique de Pleine-Fougères, sur lequel s'appuient les résultats présentés dans cet article, et une zone urbaine centrée sur la métropole de Rennes. Les principales questions de recherche portent sur le lien entre les dynamiques paysagères et celles de la biodiversité et de la qualité de l'eau. Le couplage de la géographie, en particulier avec la télédétection, de l'écologie du paysage, de l'étude des pratiques des agriculteurs et de la mise en place des politiques publiques permet de comprendre ces dynamiques. Les recherches en paléo-environnement en donnent une vision à long terme.

\section{Un exemple de système complexe: la dynamique de la biodiversité en zone bocagère}

Pour illustrer la façon dont l'interdisciplinarité permet de traiter la complexité, nous utiliserons ici les travaux sur la biodiversité du bocage. Ils sont à la base de nombreuses politiques, comme la Politique agricole commune (PAC) et ses zones d'intérêt écologiques ou encore la politique Trame verte et bleue (TVB) et ses continuités boisées. Cet article synthétise les divers résultats de recherche pour construire le lien entre la biodiversité du bocage et l'action publique (Fig. 1). En particulier, nous montrons la grande diversité des interactions entre la décision politique et les pratiques sur le terrain. Ces interactions sont source de bifurcations et de complexité.

Pour présenter la chaîne des interactions, nous partons de l'élément cible, la haie, et nous remontons vers les échelles spatiales supérieures: l'exploitation agricole, le paysage et les niveaux de construction de

\footnotetext{
$\overline{{ }^{5} \text { https://osur.univ-rennes } 1 . f r / z a-a r m o r i q u e / ~}$
} 


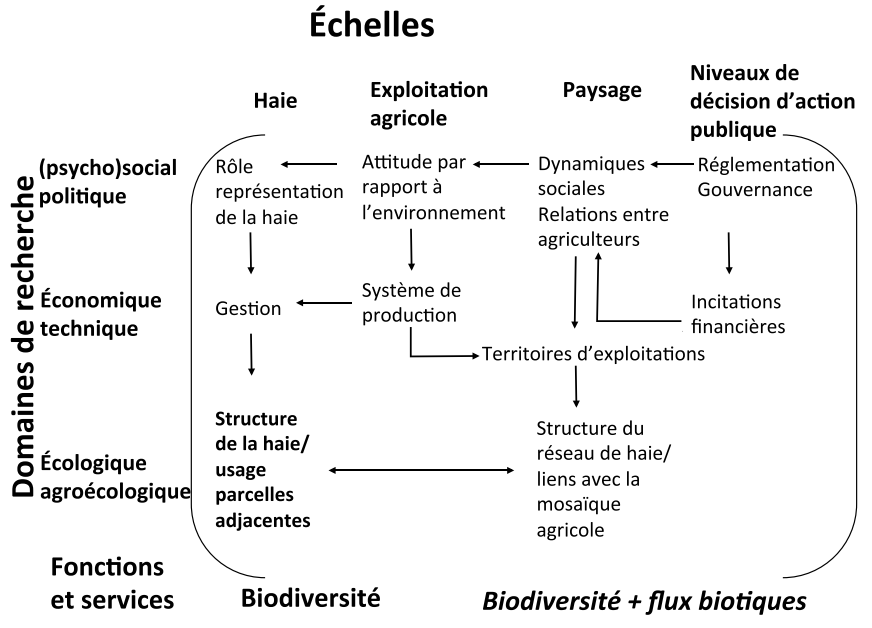

Fig. 1. Relations entre les variables des divers domaines de recherches aux échelles spatiales.

l'action publique. Notre hypothèse est que la complexité du système bocager en tant qu'entité biologique est due aux interactions entre processus de nature différentes (écologique, technique, sociale) sur une diversité d'échelles spatiales et temporelles. Ces interactions peuvent être représentées par le cadre pluri-échelles développé par Kinzig et al. (2006).

Les espèces animales et végétales présentes dans une haie dépendent de sa structure physique (présence ou non de talus et fossé) et végétale (recouvrement des strates arbustives et arborescentes) qui détermine le microclimat (Baudry et al., 2000). Si les structures physiques sont, en général, une caractéristique régionale, la structure de la végétation renvoie à la fois à une conception régionale de la haie (type d'arbre, etc.) mais aussi aux pratiques des agriculteurs. On trouve ainsi une relation entre la biodiversité des haies et, d'une part l'utilisation des parcelles adjacentes (donc des systèmes de culture), d'une autre les modes de gestion qui sont aussi liés à la diversité des exploitations agricoles (Le Coeur et al., 2002). Par ailleurs, l'écologie du paysage a montré, dès ses premiers développements, que la biodiversité des haies dépend aussi de leur place dans le paysage, en particulier dans le réseau de haies qui facilite ou non la dispersion des espèces. La haie est donc un élément d'un système sociotechnique (l'exploitation agricole) et d'un système écologique englobant (le paysage). On comprend donc que la multiplicité des facteurs de la dynamique de la biodiversité ne permet pas la construction d'un modèle déterministe, à l'échelle de la haie.

À l'échelle de l'exploitation agricole, d'autres facteurs sont à prendre en compte: le système de production qui détermine les systèmes de culture, le territoire qui façonne la localisation des systèmes de culture dans le paysage et l'attitude de l'agriculteur ou de l'agricultrice vis-à-vis des questions d'environnement
(Kergreis et Thenail, 2007). On notera que, dans cette question de rapport à l'environnement, c'est le ménage agricole qui est impliqué avec des différences marquées selon que le conjoint travaille ou non à l'extérieur de l'exploitation (Baudry et al., 1998). La mosaïque paysagère est donc formée de l'assemblage des territoires d'exploitation, ce qui n'en fait pas une structure aléatoire (Thenail et Baudry, 2004), mais son évolution est difficilement prédictible (Burel et Baudry, 1990). L'agriculteur et le ménage agricole sont dans des réseaux socioprofessionnels. Ceci génère souvent des dynamiques de groupe qui façonne, collectivement, pour partie le paysage (Capitaine, 2005). Ces groupes peuvent se saisir d'instruments de politique publique pour traiter des questions d'environnement, comme les groupements d'intérêt économique et environnemental (GIEE). Ces groupes ont, nécessairement, un ou des leaders qui les ont constitués. Or, il a été amplement montré que les problèmes malicieux, s'ils n'ont pas de solution technique optimale, peuvent être traités par une démarche de leadership (Head et Xiang, 2016). Le ou les leaders impulsent une direction qui nécessite des actions pour progresser vers un objectif partagé. Aux niveaux plus englobants, les impacts de l'action publique conçue, mise en œuvre à de multiples échelles, de la Région à l'État, l'Union européenne et les conventions internationales (e.g. OMC [Organisation mondiale du commerce]) sont aussi très importants. Tous ces collectifs font que la complexité peut être appréhendée. Elle ne condamne pas à l'impuissance si l'on sait changer l'échelle d'action et de décision (Innes et Booher, 2016).

La complexité vient également de la diversité des agriculteurs, de la diversité de leurs pratiques intraexploitation et des interactions écologiques au sein des paysages. Il n'y a donc pas de congruence entre les pratiques, les perceptions et les dynamiques écologiques aux différentes échelles. Malgré tout, il existe une cohérence au niveau d'unités paysagères constituées d'exploitations agricoles ou de fragments d'exploitations particuliers. Ces unités sont reconnaissables par leur structure et ont des biodiversités différentes (Aviron et al., 2005). Dans cette démarche, l'interdisciplinarité aide à comprendre la complexité du système. L'agronomie modélise la façon dont les agriculteurs aménagent les paysages et spatialisent leurs systèmes de culture c'est-à-dire les prairies et la mosaïque des cultures au sein de cet espace aménagé. L'anthropologie et la psychosociologie décryptent les attitudes et les comportements des agriculteurs (Javelle, 2007; Lotfi et al., 2010). Le droit et les sciences politiques analysent la construction et la mise en place de l'action publique. Mais la collaboration entre disciplines permet aussi des avancées dans l'analyse de systèmes qui peuvent être complexes comme le paysage. Ainsi, la télédétection couplée à l'écologie du paysage permet de mieux 
caractériser la structure des haies et les réseaux de haies et donc d'améliorer la compréhension du lien entre ces structures et la distribution des espèces forestières présentes dans les haies (Betbeder et al., 2015). Les pratiques de gestion sont l'autre facteur essentiel de la dynamique de la biodiversité; ce sont elles qui expliquent les variations de la composition floristique au cours du temps (Alignier et Baudry, 2015).

Ce qui vaut pour le réseau bocager qui peut constituer une trame boisée, vaut aussi pour d'autres réseaux (prairies, mosaïques de cultures, etc.) qui sont en synergie ou en antagonisme. Ainsi le réseau des prairies est utilisé par les papillons, dans ce cas, les haies peuvent constituer des barrières (Ouin et al., 2008). La mosaïque des cultures à différents stades phénologiques offre des continuités aux auxiliaires se déplaçant au sol (carabiques). Dans ce cas aussi les haies peuvent être des entraves à leur mouvement (Betbeder, 2015).

\section{Discussion}

La diversité des disciplines est indispensable pour comprendre les processus multi-échelles et, surtout, multi-domaines (écologie, technique, politique). Elle a une fonction variable selon la complexité du soussystème étudié. Cela va de l'amélioration de la compréhension des relations de cause à effet pouvant être traduites en termes de gestion (effet de la structure des haies) à la formalisation des interactions complexes, comme l'exemple donné dans la Figure 1. Nous retrouvons les deux pôles que constituent, d'un côté, les systèmes maîtrisables par des règles de gestion et, de l'autre, les systèmes complexes, multi-échelles dont la gestion est un problème malicieux sans règle optimale.

Le dispositif des zones atelier, de par son ancrage territorial et sa pérennité, permet d'aborder les systèmes complexes de façon interdisciplinaire et sur le temps long, caractéristiques nécessaires à la fois à la compréhension des processus, mais aussi au dialogue entre chercheurs ayant des questions, des méthodes et des cadres théoriques différents, qu'ils soient de disciplines elles-mêmes différentes ou semblables. Par exemple, l'écologie du paysage a emprunté l'utilisation massive de la cartographie à la géographie. Cependant, alors que la notion de gradient est centrale dans la théorie écologique comme en géographie, les écologues du paysage ont continué à utiliser des cartes représentant des mosaïques constituées de types d'éléments (bois, blé, prairie, etc.) sans prendre en compte leur diversité phénologique ou structurelle, alors que la géographie proposait depuis plusieurs années des cartes représentant des variables quantitatives permettant de distinguer des champs de blé ou de maïs les uns des autres. Il a fallu une présentation de ces potentialités en terme écologiques pour que les écologues utilisent des cartes quantitatives (Betbeder et al., 2015). Ceci a eu une conséquence importante en écologie; en utilisant des cartes qualitatives de mosaïques, la qualité des habitats restait un angle mort de l'écologie du paysage ce qui nuisait à l'articulation avec l'écologie des populations ou des communautés pour qui cette qualité est centrale.

La prise en compte des «problèmes malicieux » a mis plus de 30 ans à entrer de plain-pied dans les pratiques d'aménagement et de gestion. Il est remarquable que le concept s'applique aussi bien à la gestion de la biodiversité, du changement climatique ou la construction d'un terminal d'aéroport (Hancock, 2010). Le concept permet aussi bien de structurer une recherche interdisciplinaire qu'une action prenant en compte la multifonctionnalité des territoires.

\section{Remerciements}

La zone atelier Armorique bénéficie d'un financement récurent de l'institut écologie et environnement du CNRS et de l'Inra. Les projets ayant permis les travaux cités dans cet article ont été financés par divers programmes du ministère de l'Environnement et par des programmes européens. Merci aux membres du comité d'animation de la zone atelier Armorique pour leurs commentaires.

\section{Références}

Alignier A., Baudry J., 2015. Changes in management practices over time explain most variation in vegetation of field margins in Brittany, France. Agriculture, Ecosystems \& Environment, 211, 164-172.

Aviron S., Burel F., Baudry J., Schermann N., 2005. Carabid assemblages in agricultural landscapes: impacts of habitat features, landscape context at different spatial scales and farming intensity. Agriculture, Ecosystems \& Environment, 108, 3, 205-217.

Baudry J., Jouin A., Thenail C., 1998. La diversité des bordures de champ dans les exploitations agricoles de pays de bocage. Études et Recherches sur les Systèmes Agraires et le développement, 31, 117-134, https://hal.archivesouvertes.fr/hal-01231559.

Baudry J., Bunce R.G.H., Burel F., 2000. Hedgerow: an international perspective on their origin, function, and management. Journal of Environmental Management, 60, 7-22.

Betbeder J., 2015. Évaluation des données de télédétection pour l'identification et la caractérisation des continuités écologiques. Thèse de doctorat, Rennes, Université Rennes 2.

Betbeder J., Hubert-Moy L., Burel F., Corgne S., Baudry J., 2015. Assessing ecological habitat structure from local to landscape scales using synthetic aperture radar. Ecological Indicators, 52, 545-557. 
Burel F., Baudry J., 1990. Structural dynamic of a hedgerow network landscape in Brittany France. Landscape Ecology, 4, 4, 197-210.

Capitaine M., 2005. Organisation des territoires des exploitations agricoles. Impact du recours à des collectifs d'action : la conduite de chantiers de récolte en CUMA. Thèse, Nancy, Institut Polytechnique de Lorraine, http://docnum.univlorraine.fr/public/INPL/2005_CAPITAINE_M.pdf.

Carpenter S.R., Armbrust E.V., Arzberger P.W., Stuart Chapín F., Elser J.J., Hackett E.J., Ives A.R., Kareiva P.M., Leibold M.A., Lundberg P., Mangel M., Merchant N., Murdoch W. W., Palmer M.A., Peters D.P.C., Pickett S.T.A., Smith K.K., Wall D.H., Zimmerman A.S., 2009. Accelerate synthesis in ecology and environmental sciences. Bioscience, 59, 8, 699-701.

De Longe M.S., Miles A., Carlisle L., 2016. Investing in the transition to sustainable agriculture. Environmental Science \& Policy, 55, Part 1, 266-273.

Hancock D., 2004. Tame problems and wicked messes: choosing between management and leadership options. The RMA Journal, 38-42.

Hancock D., 2010. Tame, messy and wicked risk leadership, Farnam, UK, Gower Publishing Ltd.

Head B.W., Xiang W.N., 2016. Working with wicked problems in socio-ecological systems: more awareness, greater acceptance, and better adaptation. Landscape and Urban Planning, 154, 1-3.

Innes J.E., Booher D.E., 2016. Collaborative rationality as a strategy for working with wicked problems. Landscape and Urban Planning, 154, 8-10.

Javelle A., 2007. Perceptions de la biodiversité par des agriculteurs sur une zone atelier du nord-est de la Bretagne et évaluation de leur rencontre avec des chercheurs en environnement, ou la main et le stylo. Thèse de doctorat, Rennes, Université Rennes 1.

Jollivet M., 1992. Pluridisciplinarité, interdisciplinarité et recherche finalisée ou des rapports entre sciences, techniques et sociétés. In M. Jollivet (Dir.), Sciences de la nature. Sciences de la société. Les passeurs de frontières, Paris, CNRS éditions, 519-535, http://books.openedition. org/editionscnrs/4216?lang=fr.

Kergreis S., Thenail C., 2007. Attitudes et comportements des agriculteurs vis-à-vis de la gestion des bordures de champ en paysage de bocage. In Antoine A., Marguerie D. (Dir.), Bocage et Société, Rennes, Presses Universitaires de Rennes, 385-395.

Kinzig A.P., Ryan P.A., Etienne M., Allison H.E., Elmqvist T., Walker B.H., 2006. Resilience and regime shifts: assessing cascading effects. Ecology and Society, 11, 1, 20, https:// ecologyandsociety.org/vol11/iss1/art20/.

Laurent C., Baudry J., Berriet-Solliec M., Kirsch M., Perraud D., Tinel B., Trouvé A., Allsopp N., Bonnafous P., Burel F., 2009. Pourquoi s'intéresser à la notion d' « evidence-based policy »?, Revue Tiers Monde, 4, 200, 853-873, https:// cairn.info/revue-tiers-monde-2009-4-page-853.htm.

Le Coeur D., Baudry J., Burel F., Thenail C., 2002. Why and how we should study field boundaries biodiversity in an agrarian landscape context. Agriculture, Ecosystems \& Environment, 89, 1-2, 23-40.

Liu J., Dietz T., Carpenter S.R., Alberti M., Folke C., Moran E., Pell A.N., Deadman P., Kratz T., Lubchenco J., Ostrom E., Ouyang Z., Provencher W., Redman C.L., Schneider S. H., Taylor W.W., 2007. Complexity of coupled human and natural systems. Science, 317, 5844, 1513-1516, http:// science.sciencemag.org/content/317/5844/1513.full.

Lotfi A., Javelle A., Baudry J., Burel F., 2010. Interdisciplinary analysis of hedgerow network landscapes' sustainability. Landscape Research, 35, 4, 415-426.

Maass M., Balvanera P., Baudry J., Bourgeron P., Dick J., Equihua M., Forsius M., Halada L., Krauze K., Nakaoka M., Orenstein D.E., Parr T., Redman C.L., Rozzi R., SantosReis M., Swemmer T., Vădineanu A., 2016. Changes in biodiversity and trade-offs among ecosystem services, stakeholders and components of well-being: the contribution of the ILTER to PECS. Ecology and Society, 21, 3, 31, https://ecologyandsociety.org/vol21/iss3/art31/.

Ouin A., Martin M., Burel F., 2008. Agricultural landscape connectivity for the meadow brown butterfly (Maniola jurtina). Agriculture, Ecosystems \& Environment, 124, 3-4, 193-199.

Rittel H.W., Webber M.M., 1973. Dilemmas in a general theory of planning. Policy sciences, 4, 2, 155-169.

Thenail C., Baudry J., 2004. Variation of farm spatial land use pattern according to the structure of the hedgerow network (bocage) landscape: a case study in northeast Brittany. Agriculture, Ecosystems \& Environment, 101, 1, 53-72.

Citation de l'article : Baudry J., Alignier A., Thomas Z., 2017. Interdisciplinarité et représentation de la complexité des systèmes socio-écologiques: recherches sur la zone atelier Armorique. Nat. Sci. Soc. 25, S50-S54. 